\title{
Angiolymphoid Hyperplasia with Eosinophilia: A Study of Two Cases
}

\author{
Pektas $\mathrm{SD}^{1} *$, Dere $\mathrm{Y}^{2}$, Semra Hancer $\mathrm{H}^{1}$, Kutucularoglu $\mathrm{EU}^{3}$ and \\ Dogan $G^{1}$ \\ ${ }^{1}$ Department of Dermatology, Mugla SitkiKocman University Faculty of Medicine, \\ Turkey \\ ${ }^{2}$ Department of Pathology, Mugla SitkiKocman University Faculty of Medicine, Turkey \\ ${ }^{3}$ Department of Dermatology, Aydin State Hospital, Turkey
}

\section{Case Report}

Volume 2 Issue 3

Received Date: July 11, 2017

Published Date: July 29, 2017

DOI: $10.23880 /$ cdoaj16000128

*Corresponding author: Suzan Demir Pektas, MD, Department of Dermatology, Mugla Sitki Kocman University Faculty of Medicine 48000, Mugla, Turkey, Tel: +90 252211 5219, Fax: +90 312 3116768; E-mail: suzandpektas@gmail.com

\section{Abstract}

Objective: Angiolymphoid hyperplasia with eosinophilia (ALHE), is presented by multiple pink or red papules and nodules on the head, neck, preauricular area and anterior hairline. Although the lesions are usually asymptomatic, they may rarely accompany pain, pulsation, itching and spontaneous bleeding when is pressed on. The etiopathogenesis of ALHE is related to environmental, allergic, hormonal and immunological factors. Here, the purpose of our study is to review the literature and to observe this vasculo-proliferative disease by reporting two case of ALHE associated with an allergic response, one pregnant one man.

Case Report: High serum IgE levels and eosinophilia-rich infiltration on histopathological examination were showed in both of our cases. Additionally, IgE antibody was positive in immunohistochemical examination. These findings support the fact that ALHE lesions may be associated with an allergic response in both of our cases.

Conclusion: ALHE, which has a lot of questions in its etiopathogenesis, this disease will be better understood by largescale studies in a large number of studies, so that the appropriate treatment method will be chosen and the recurrences after treatment will be less

Keywords: ALHE; High serum Ig E levels; Positive IgE stain; Pregnant; Man

\section{Introduction}

Angiolymphoid hyperplasia with eosinophilia (ALHE), first described by Wells and Whimster in 1969, is a vasculo-proliferative disease [1]. It affects women between 20 and 40 years of age. ALHE is presented by multiple pink or red papules and nodules on the head, neck, preauricular area and anterior hairline. Although the lesions are usually asymptomatic, they may rarely accompany pain, itching and spontaneous bleeding when pressed on. The etiopathogenesis of ALHE is related to environmental, hormonal and immunological factors [2]. Here, the purpose of our study is to review the literature and to present this vasculo-proliferative disease by 
reporting two case of ALHE, one pregnant woman and one man.

\section{Case Report}

\section{Case-1}

A 28-year-old woman presented to the dermatology clinic slightly pruritic, painful, recurrent bleeding red-blue lumps over scalp. She reported that her complaint had started when she was 6-month-pregnant and an increase in the number and size of bulges in course of time. On dermatologic examination, erythematous, endured, clustered, $0,5-2 \mathrm{~cm}$ size nodules on the left occipital region were seen (Figure 1A). Physical examination and blood tests were normal except total IgE: 952,7 kIU/L ( $\mathrm{N}: 0-165)$. Cranial magnetic resonance angiography in the left occipital region was observed dilated tortuous occipital arteries in skin and subcutaneous tissue and thickening of the skin. An incisional skin biopsy was performed with differential diagnosis of ALHE, Kimura's disease and lyphoma. ALHE was diagnosed with clinical and histopathological findings. One session cryotherapy was performed to lesions but the patient did not apply for the second session. The patient reappeared 2 months after giving birth, when her lesions grew gradually and increased frequency of bleeding. She was consulted to cardiovascular surgery department.
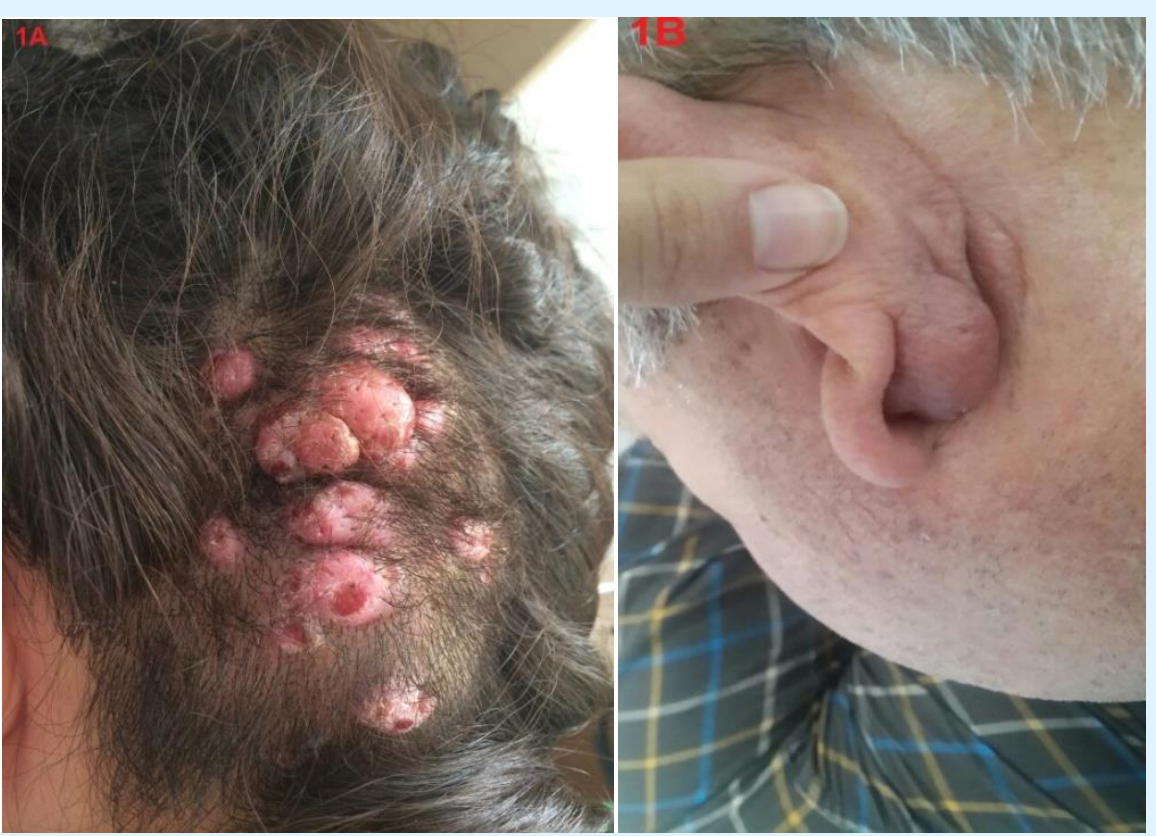

Figure 1: A: In Case 1, has a diameter of $0.5 \times 2 \mathrm{~cm}$, a grubed, erythematous, and some erode nodosites on the left occipital region; $\mathrm{B}$ : In case 2 , behind the left ear $0.8 \times 2 \mathrm{~cm}$ diameter one nodosity.

\section{Case-2}

A 64-year-old man presented with blue-red bumps and severe itching behind both ears. The patient pointed out that initially developed an itchy bulge behind his right ear 3 months earlier, and developed a lesion behind the left ear 1 month after that and an increase in the size and shape of the lesions over time. We noted $0,8 \times 2 \mathrm{~cm}$ diameter nodosity behind both ears on dermatologic examination (Figure 1B). No abnormal findings were found on physical examination. Laboratory tests were normal except total IgE $>6000 \mathrm{kIU} / \mathrm{L}$ (N:0-165). An incisional skin biopsy was performed for ALHE, Kimura's disease and histiocytosis. ALHE was diagnosed with clinical and histopathological findings. Patient refused treatment but surgical excision was performed to the patient six months later because of increased itching. No recurrence was observed six-month follow up.

\section{Histological findings}

In both cases, the histopathologic features were similar. Histopathological examination revealed proliferation of vessels as well as inflammatory infiltrate in which eosinophils were dominant, in the dermis and subcutaneous tissue in case 1 (Figure 2A) . In case 2, vacuolated endothelial cell and vessel proliferation, lymphoid cell aggregates associated with dilate vessels and eosinophilic infiltration was detected in papillary dermis (Figure 2B). 
Dilated vascular structures and endothelial cells were CD34 (+) and CD31 (+) in addition to the mixed reactive staining of CD3 and CD 20 in the inflammatory areas by immunohistochemistry of both cases. Estrogen and progesterone receptor were (-) and Ig E was (+) in both cases (Figure 2C-D).
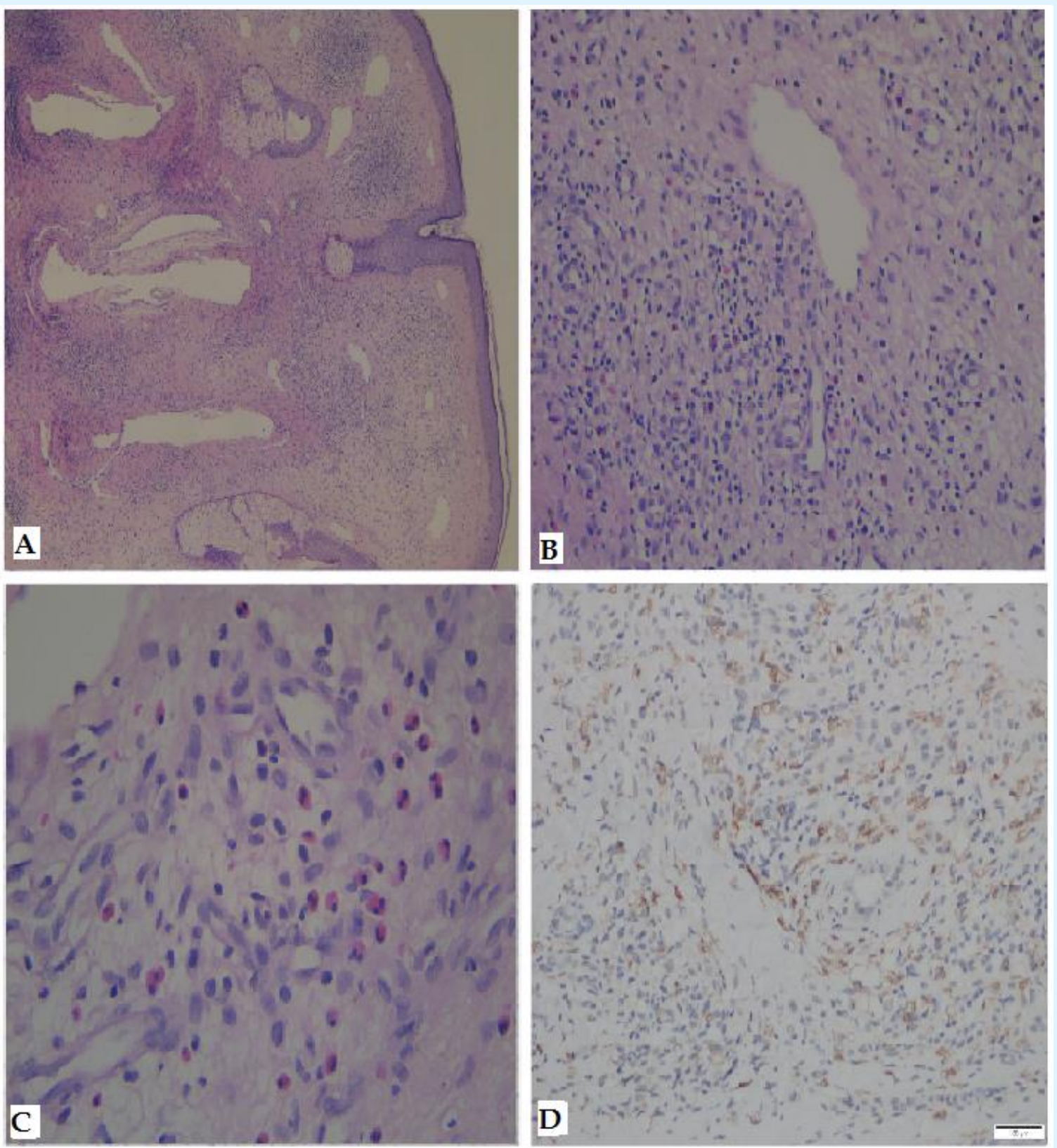

Figure 2: A: In case 1, lobular vasculature proliferation in which the inflammatory component is accompanied and eosinophils predominate in the dermis and subcutaneous area HE, X40.

B: In case 2, vacuolated endothelial cell and vessel proliferation, dilate vessels, lymphoid cell aggregates and eosinophilic infiltration in the papillary dermis, HE, X100.

C: Numerous eosinophils located perivascular region, HE, X400.

D: IgE staining was positive, DAB, X200 


\section{Discussion}

Although the etiopathogenesis of ALHE is not fully known, it is mainly related to trauma, hyperestrogenemia, infectious agents, environmental factors (insect bite), atopy, disorders in the immunological mechanism [2,3]. In the literature, it has been reported cases associated with hyper estrogenemic conditions (pregnancy, use of oral contraceptives) [4-7]. Moy, et al. detected the expression of estrogen and progesterone receptors in lesions at two cases in their study [4]. Hollo P, et al. did not define increase in the amount of estrogen and progesterone receptors in the ALHE lesion during pregnancy [5]. Estrogens and progesterone staining were negative in both of our cases. In our first case, the increase in the number and size lesions that developed during the $6^{\text {th }}$ gestational month occured and also continued after the pregnancy. Besides in the second case the patient is male and the negative detection of estrogen and progesterone staining in both lesions suggests that another factor may play a role in the development of ALHE.

One of the factors accused in the etiopathogenesis of ALHE is the IgE-mediated hypersensitivity reaction. Wangs SX, et al. reported that peripheral eosinophilia and high serum Ig E levels in three cases and revealed significant eosinophil in histopathological examination so they detected that may have an allergic role in the etiopathogenesis of ALHE [6]. Similarly, Von den Driesch $\mathrm{P}$, et al. reported that $\mathrm{T}$ helper lymphocytes, monocytes, eosinophil, CD1 + dendritic cells and mast cell infiltration in a case of ALHE. Also they detected IgE surface binding on these cells [7].

It has been suggested that ALHE may develop a reactive angiogenic response to $\mathrm{CD} 4+\mathrm{T}$ cell mediated lymphoproliferative disorder rather than a reactive inflammatory vascular lesion [3]. High serum IgE levels and eosinophilia-rich infiltration on histopathological examination were showed in both of our cases. Additionally, IgE antibody was positive in immunohistochemical examination. These findings support the fact that ALHE lesions may be associated with an allergic response in both of our cases.

There are a large number of benign and malignant vascular disease in ALHE's differential diagnosis. These disease are mainly Kimura disease (KD), fibrous histiocytoma, eruptive pyogenic granuloma, cavernous hemangioma, squamous papilloma, sarcoidosis, keratoacanthoma, basillar angiomatosis, cavernous hemangioma, and malignant vascular lesions [8,9]. KD affects Asian men in the age of 10-30 years. It is generally seen as painless subcutaneous nodules unilaterally located in head and neck, and diffuse lymphadenopathy. ${ }^{8}$ Histiocytic endothelial cells that characteristic of ALHE are rare in KD [10]. In the histopathological examination of the lesions in both of our cases; germinal-centered lymphoid follicles and fibrosis were not observed. In addition, absence of eosinophilia, lymph node involvement, salivary gland and kidney involvement supported the diagnosis of ALHE in two of our cases.

There are many treatment options for ALHE treatment, including surgical excision, cryosurgery, laser, corticosteroid, electrocoterization, interferon alfa-2a, isotretinoin and vinblastine [11,12]. Another option is to follow up the lesion 3-6 months for the possibility of spontaneous regression [2]. Surgical excision which has a high success rate is widely used $[10,11]$. In our first case, surgical excision was performed because of bleeding and itching. In our second case, who did not accept the treatment at the initial stage, surgical excision was performed on the absence of regression of lesion and increased complaint of itching.

Despite treatment, it has been reported recurrence of the disease approximately $30 \%$ of ALHE cases but no metastasis [10]. No recurrence was observed at 6 months follow-up after surgical excision in both of our cases.

\section{Conclusion}

We believe that ALHE, which has a lot of questions in its etiopathogenesis, will be better understood by largescale studies in a large number of studies, so that the appropriate treatment method will be chosen and the recurrences after treatment will be less.

What is already known on this topic: The etiopathogenesis of ALHE is mainly related to trauma, hyperestrogenemia, infectious agents, environmental factors (insect bite), atopy, disorders in the immunological mechanism. In many studies, role of IgE in ALHE lesion has been investigated and controversial results have been obtained.

What this study adds: High serum IgE levels and eosinophilia-rich infiltration on histopathological examination were showed in both of our cases. Additionally, IgE antibody was positive in immunohistochemical examination. These findings support the fact that ALHE lesions may be associated with an allergic response in both of our cases. IgE may play a major role in the development and prognosis of ALHE. ALHE has a lot of questions in its etiopathogenesis, will be better understood by large-scale studies in a large number of studies. 


\section{Clinical Dermatology Open Access Journal}

Author's contributions: Conception and design: Pektas SD, Dogan G; Acquisition, analysis and interpretation of data: Pektas SD, Dogan G, Dere Y, Hancer HS and Kutucularoglu; Drafting the article: Pektas SD; Revising it critically for important intellectual content: Pektas SD, Dogan G, Dere Y; Approved final version of the manuscript: Pektas SD, Dogan SD.

\section{References}

1. Wells GC, Whimster IW (1969) Subcutaneous angiolymphoid hyperplasia with eosinophilia. $\mathrm{Br} \mathrm{J}$ Dermatol 81(1): 1-14.

2. Shenefelt PD, Rinker M, Caradonna S (2000) A case of angiolymphoid hyperplasia with eosinophilia treated with intralesional interferon alfa-2a. Arch Dermatol 136(7): 837-839.

3. Kimura $\mathrm{Y}$, Tsutsumi T, Kuroishikawa $\mathrm{Y}$, Kishimoto $\mathrm{S}$ (2003) Angiolymphoid hyperplasia with eosinophilia arising from the facial artery. J Laryngol Otol 117(7): 570-573.

4. Moy RL, Luftman DB, Nguyen QH, Amenta JS (1992) Estrogen receptors and the response to sex hormones in angiolymphoid hyperplasia with eosinophilia. Arch Dermatol 128(6): 825-828.

5. Hollo P, Marschalko M, Sikos G, Harsing J, Horvath A (2005) Angiolymphoid hyperplasia with eosinophilia in pregnancy. J Eur Acad Dermatol Venereol 19(5): 645-646.
6. Wang SX, Zou WZ, Lü JC, Su T, Zhou FD, et al. (2007) Angiolymphoid hyperplasia with eosinophilia involving the kidney: a report of three cases with literature review. Zhonghua Nei Ke Za Zhi 46(10): 827-830.

7. Von den Driesch P, Gruschwitz M, Schell H, Sterry W (1992) Distribution of adhesion molecules, IgE, and CD23 in a case of angiolymphoid hyperplasia with eosinophilia. J Am Acad Dermatol 26(5 Pt 2): 799804.

8. Busquets AC, Sánchez JL (2006) Angiolymphoid hyperplasia with eosinophilia induced by trauma. International Journal of Dermatology 45(10): 12111214.

9. Marcum CB, Zager JS, Belongie IP, Messina JL, Fenske NA (2011) Profound proliferating angiolymphoid hyperplasia with eosinophilia of pregnancy mimicking angiosarcoma. Cutis 88(3): 122-128.

10. Guo R, Gavino AC (2015) Angiolymphoid hyperplasia with eosinophilia. Arch Pathol Lab Med. 139(5): 683686.

11. Baghestani S, Firooz A, Ghazisaidi MR (2011) A refractory case of angiolymphoid hyperplasia with eosinophilia successfully treated by surgery. J Dermatolog Treat 22(1): 49-51.

12. El Sayed F, Dhaybi R, Ammoury A, Chababi M (2006) Angiolymphoid hyperplasia with eosinophilia: efficacy of isotretinoin? Head Face Med 2: 32-36. 